FERMILAB-Pub-01/043-T

October, 2000

\title{
Gauge Invariant Effective Lagrangian for Kaluza-Klein Modes
}

\author{
Christopher T. Hill ${ }^{1}$ \\ Stefan Pokorski ${ }^{1,2}$ \\ Jing Wang ${ }^{1}$ \\ ${ }^{1}$ Fermi National Accelerator Laboratory \\ P.O. Box 500, Batavia, Illinois 60510, USA* \\ 2 Institute for Theoretical Physics \\ Hoza 69, 00-681, Warsaw, Poland
}

October 24, 2018

\begin{abstract}
We construct a manifestly gauge invariant Lagrangian in $3+1$ dimensions for $N$ Kaluza-Klein modes of an $S U(m)$ gauge theory in the bulk. For example, if the bulk is $4+1$, the effective theory is $\Pi_{i=1}^{N+1} S U(m)_{i}$ with $N$ chiral $(\bar{m}, m)$ fields connecting the groups sequentially. This can be viewed as a Wilson action for a transverse lattice in $x^{5}$, and is shown explicitly to match the continuum $4+1$ compactified Lagrangian truncated in momentum space. Scale dependence of the gauge couplings is described by the standard renormalization group technique with threshold matching, leading to effective power law running. We also discuss the unitarity constraints, and chiral fermions.
\end{abstract}

*e-mail: hill@fnal.gov, jingw@fnal.gov, Stefan.Pokorski@fuw.edu.pl 


\section{Introduction}

It is widely believed that the main low energy signature of extra dimensions is the appearance of the tower of Kaluza-Klein (KK) modes [1]. For example, if QCD lived in the bulk, experimentalists would see massive spin-1 degenerate color octet vector bosons (colorons) appearing at large mass scales corresponding to (inverse) compactification scales. As these new massive KK particles begin to emerge in accelerator experiments, we might ask how would we describe them in an effective four-dimensional renormalizeable Lagrangian that is an extension of QCD, without an a priori knowledge of the existence of extra dimensions? The main goal of the present paper is to give a manifestly gauge invariant effective Lagrangian description of KK modes in $3+1$ dimensions.

It is important to realize at the outset that there is an implicit dynamical assumption underlying a theory with extra-dimensions and KK modes. This is the assumption that there is a meaningful separation of scales between the compactification scale, $M_{c} \sim 1 / R$ and the "string" or "fundamental scale" $M_{s}$ at which the extra-dimensional theory breaks down as a perturbative local field theory. To have $N>>1 \mathrm{KK}$ modes in a $4+1$ theory we require $M_{s} / M_{c} \sim N>>1$. It is not obvious how such a separation of scales occurs in the theory (It involves soft mass scales in the radion potential that somehow remain isolated from $M_{s}$ ). Can it occur naturally or does it require fine-tuning? Such a hierarchy requires strong coupling at the high energy scale $M_{s}$. We will assume, as do all extra-dimensional models, that we have such a hierarchy, and return to this issue in Section 6.

Having engineered a hierarchy with $N>1 \mathrm{KK}$ modes, by analogy with critical behavior in a second order phase transition in condensed matter physics, there should exist a wide range, or universality class, of theories that have identical behavior in the infra-red, but are radically different in detail at the scale $M_{s}$. In the present paper we exploit universality. We treat the physics at $M_{s}$ not as a "string theory," but rather as a "transverse lattice gauge theory" [2]. For us, the normal 3+1 dimensions of space-time are continuous, but the extra dimensions are latticized (nothing prevents us from adopting a full lattice theory, but it is convenient for our presnt purposes to use the transverse lattice). This theory will have a well-defined finite short-distance behavior for arbitrarily

large coupling and will be manifestly gauge invariant, reflecting the full gauge invariance of the higher dimensional theory. It will have the same infra-red behavior as the usual KK-mode description, but will illuminate how the gauge invariance is maintained.

As a result, we understand something implicitly puzzling about KK modes. Longitu- 
dinal KK mode scattering is essentially the scattering of Nambu-Goldstone bosons in a nonlinear chiral Lagrangian. As such it violates perturbative unitarity, i.e., there is a LeeQuigg-Thacker bound on the applicability of the theory [3]. We will see that this happens at, none other than, the scale $M_{s}$ in our effective Lagrangian. This is not surprising, since the parent $D=5$ theory has a dimensional coupling constant $g_{0}$ (with dimension $M^{-1 / 2}$ ) and is expected to violate perturbative unitarity when $s \gtrsim M_{s} / \alpha_{0}$. This indeed translates into the unitarity bound $s \lesssim 4 \pi v^{2}$ for longitudinal gauge boson scattering in our effective $3+1$ theory.

The main reason for desiring an approach such as this is that it is difficult to treat nonabelian gauge theories in loop expansions with momentum space cut-offs. Normally, the momentum space cut-off is not compatible with gauge invariance, and this causes the loop expansion to become non-gauge invariant. However, the usual treatment of extra-dimensional gauge theories involves a truncation on KK modes, which is a de facto momentum space cut-off. With gauge fields in the bulk, a $d+1$ theory with $d>3$ has infinitely more gauge invariance than the $3+1$ theory since there is more space in which to perform local gauge transformations. Clearly the gauge invariance of $3+1 \mathrm{QCD}$ must be maintained, but how does the expanding local gauge invariance of the theory manifest itself as the extra dimension begins to open up with the emergence of KK modes? How does the power-law running of the coupling constant emerge and what is the correct renormalization group for such a description?

\section{Manifestly Gauge Invariant Effective Lagrangian}

The KK modes of the vector bosons of QCD, i.e., the colorons, are heavy matter fields and must transform linearly under the adjoint representation of $S U(3)$ (in contrast to the zero-mode gluon which transforms nonlinearly by the Yang-Mills gauge transformation). References [4] have argued that vector fields in linear adjoint representations of a local gauge group $S U(m)$ will always contain a "hidden" local symmetry, which is a copy of $S U(m)$. The gluon plus one massive octet vector multiplet corresponds to the local symmetry $S U(m) \times S U(m)$, each factor having the same coupling constant (our present discussion is classical; we'll worry about running couplings below). This is broken diagonally by an effective Higgs field, $\Phi$, which transforms as a $(\bar{m}, m)$, to a local $S U(m)$ and an $S U(m)$ global symmetry. Only the chiral components of $\Phi$ are relevant here so we can replace $\Phi \rightarrow v \exp \left(\phi^{a} \lambda^{a} / 2 v\right.$ ) (see footnote [1]). The $\phi^{a}$ are eaten to give the coloron 
mass. Hence, in describing one massive octet this way it is the low energy hidden local symmetry due to the spontaneous breaking that reflects the expanded gauge invariance of the extra-dimensional theory as the space of the extra dimension is opening up.

As experiments go to higher energies, one starts to see more KK massive gauge bosons. It is obvious that one requires more "hidden" local $S U(3)$ symmetries and more Higgs fields as in the previous case to construct an effective Lagrangian to describe these massive gauge bosons. Hence, we propose that the effective Lagrangian for the first $n \mathrm{KK}$ modes would contain $N+1(N \gg n) S U(3)$ 's with $N \Phi$ 's. The interconnections between the gauge symmetries and the Higgs could become completely arbitrary, and resolve into different hydrocarbon-like chain molecules.

We might guess that the simplest linear interconnection for $N$ modes having $\Phi_{i} \subset$ $\left(\overline{3}_{i}, 3_{i+1}\right)$ is somehow relevant. We'll follow the organic chemistry nomenclature and call this an "aliphatic" $\left(S U(3)^{N+1}, \Phi^{N}\right)$ model. The Lagrangian for this scheme is:"

$$
\mathcal{L}=-\frac{1}{4} \sum_{i=0}^{N} F_{i \mu \nu}^{a} F^{i \mu \nu a}+\sum_{i=1}^{N} D_{\mu} \Phi_{i}^{\dagger} D^{\mu} \Phi_{i}
$$

in which the covariant derivative is defined as $D_{\mu}=\partial_{\mu}+i g_{L} A_{\mu}^{a} T^{a}, g_{L}$ is the dimensionless gauge coupling constant that is equal for all of the $S U(3)$ symmetries and $T^{a}$ are the generators of the gauge symmetry where $a$ is the color index. Note that the fact that $g_{L}$ is common for all the gauge groups is a key constraint and would be to the experimentalist in $3+1$ evidence of the extra-dimensions. Upon substituting,

$$
\Phi_{i} \rightarrow v \exp \left(i \phi_{i}^{a} \lambda^{a} / 2 v\right)
$$

the $\Phi$ kinetic terms lead to a mass matrix for the gauge fields:

$$
\sum_{i=1}^{N} \frac{1}{2} g_{L}^{2} v^{2}\left(A_{(i-1) \mu}^{a}-A_{i \mu}^{a}\right)^{2}
$$

This mass matrix has the structure of a nearest neighbor coupled oscillator Hamiltonian. We can diagonalize the mass matrix to find the eigenvalues (which corresponds to the

\footnotetext{
${ }^{1} \mathrm{~A}$ renormalizable potential can be constructed for the Higgs fields,

$$
V\left(\Phi_{j}\right)=\sum_{j=1}^{N}\left[-M^{2} \operatorname{Tr}\left(\Phi_{j}^{2}\right)+\lambda_{1} \operatorname{Tr}\left(\Phi_{j}^{4}\right)+\lambda_{2} \operatorname{Tr}\left(\Phi_{j}^{2}\right)^{2}+M^{\prime} \operatorname{det}\left(\Phi_{j}\right)\right],
$$

We can always arrange the parameters in the potential such that the diagonal components of each $\Phi_{j}$ develop a vacuum expectation value $v$, and the Higgs and $U(1)$ PNGB are heavy.
} 
dispersion relation for the coupled oscillator-system):

$$
M_{n}=\sqrt{2} g_{L} v \sin \left[\frac{\gamma_{n}}{2}\right] \quad \gamma_{n}=\frac{n \pi}{N+1}, \quad n=0,1, \ldots, N
$$

Thus we see that for small $n$ this system has a KK tower of masses given by:

$$
M_{n} \approx \frac{g_{L} v \pi n}{\sqrt{2}(N+1)} \quad n<<N
$$

and $n=0$ corresponds to the zero-mode gluon.

To match on to the spectrum of the KK modes, we require

$$
\frac{g_{L} v}{\sqrt{2}(N+1)}=\frac{1}{R}
$$

Hence, the aliphatic system with $S U(3)^{N+1}$ and $N \Phi_{i}$ provides a gauge invariant description of the first $n \mathrm{KK}$ modes by generating the same mass spectrum. It is thus crucial to examine the interactions from the aliphatic model.

In a geometric picture, the aliphatic model corresponds to a "transverse lattice" description of a full $4+1$ gauge theory [2]. We construct a transverse lattice in the $x^{5}$ dimension where the lattice size is $R$ and short-distance lattice cut-off is $a$, so $N=R / a$. This is a foliation of $N+1$ parallel branes, each spaced by a lattice cut-off $a$ (Fig.(1)). On the $i$ th brane we have an $S U(m)$ gauge theory denoted by $S U(m)_{i}$. The $S U(m)_{i}$ automatically have a common coupling constant $g$. Each brane $S U(m)_{i}$ theory can be viewed as predefined in the continuum limit of a fine-grained Wilson plaquette action, and a hypothetical $3+1$ lattice spacing $a_{4}$. The lattice spacing in the $x^{5}$ dimension can be viewed as relatively coarse with $a>>a_{4}$ [2].

The theory thus has $N$ links in the $x^{5}$ direction that are continuous functions of $x_{\mu}$. These correspond to the continuum limit Wilson lines:

$$
\Phi_{n}\left(x^{\mu}\right)=\exp \left[i g_{0} \int_{n a}^{(n+1) a} d x^{5} A_{5}\left(x^{\mu}, x^{5}\right)\right] \rightarrow \exp \left[i g_{0} a A_{5}\left(x^{\mu},\left(n+\frac{1}{2}\right) a\right)\right]
$$

The $N \Phi_{n}$ therefore transform as an $(\bar{m}, m)$ representation of $S U(m)_{n} \times S U(m)_{n+1}$ as in the aliphatic model (straddling the nearest neighbor $S U(m)_{n}$ and $S U(m)_{n+1}$ gauge groups). $\Phi_{n}$ is a unitary matrix and may be parameterized as in eq.(1.3). The theory is a spline approximation to the configurations in the continuum $x^{5}$ dimension. 


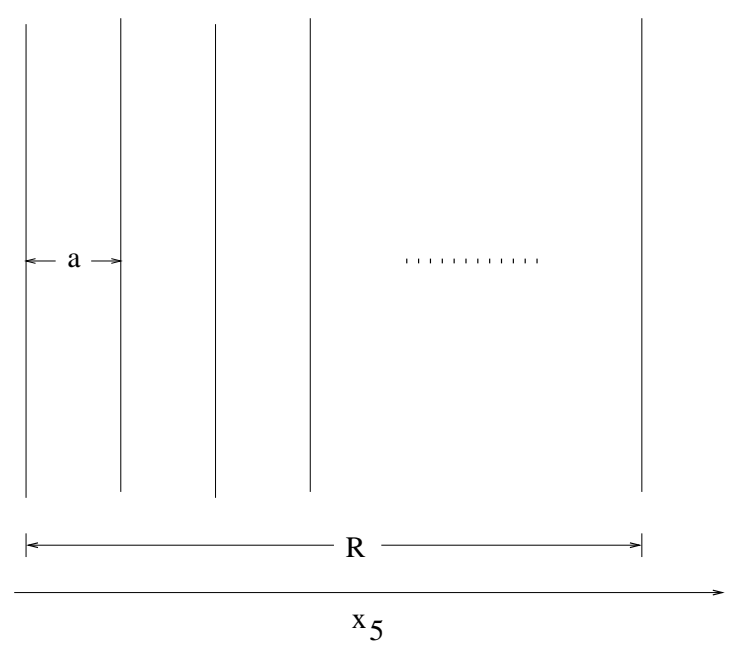

Figure 1: The geometric interpretation for the aliphatic model as a transverse lattice in the $x^{5}$ dimension with continuum theory in $4+1$. The number of branes in the foliation is $N+1=(R / a)+1$.

\section{Compare the Continuum Theory}

(i) Definition of the Continuum Theory

A $d+1(d>3)$ field theory becomes ill-defined at energy scale $M_{s}>>1 / R$. Presumably it matches onto a string theory at $M_{s}$, and we usually refer to $M_{s}$ as the "string scale." While the exact structure of the theory on scales $\mu \sim M_{s}$ is unknown, its symmetries, e.g., local gauge invariance, must remain intact at lower scales. A continuum $d+1$ Yang-Mills Lagrangian gives a valid description at scales below $M_{s}$.

A Wilson transverse lattice Lagrangian is a reasonable candidate for a well-defined short distance definition of the nonperturbative higher dimensional theory. This manifestly preserves local gauge invariance and permits, in principle, a nonperturbative treatment. How, then, does the aliphatic $\left(S U(3)^{N+1}, \Phi^{N}\right)$ model match in detail to the perturbative $4+1$ continuum theory at lower energies?

We define the continuum theory in $4+1$ and expand in modes in the compact $x^{5}$. We truncate this theory after $N$ terms. Now, momentum space truncations in Yang-Mills theories are notoriously awkward at best. The expansion is usually done in a particular gauge. Then, with truncation of the theory in momentum space we lose track of the full gauge invariance of the theory. However, we will see, remarkably, that this truncation can be matched identically onto the aliphatic theory which is manifestly gauge invariant. 
Since the aliphatic model is manifestly gauge invariant and renormalizable, various field theoretical questions can be given precise formulation. One of them is the running of the coupling constants, which at one loop level qualitatively agrees with the results of Dienes, et al. [5] and Dobrescu et al. [6]. But in our formulation it can be systematically calculated to any required degree of accuracy.

First, we consider a simple well-defined compactification scheme. We define QCD in 4+ 1 dimensions between two parallel branes. 2]. The branes are respectively located at I: $x^{5}=$ $R_{I}=0$ and II: $x^{5}=R_{I I}=R$, with a constant inter-brane separation $R$. The covariant derivative is defined as $D_{M}=\partial_{M}+i g_{0} \hat{A}_{M}^{a} T^{a}$, with field strengths $i g_{0} \hat{F}_{M N}=\left[D_{M}, D_{N}\right]$, where the canonical mass dimension of the vector potential $\hat{A}_{M}$ in $4+1$ dimensions is $3 / 2$, and the coupling constant $g_{0}$ must therefore have dimension $-1 / 2$.

The five-dimensional theory is locally gauge invariant but non-renormalizable. In addition to the compactification radius $R$, it is defined by the fundamental short-distance cut-off scale $M_{s}$. It is then natural to define a dimensionless $g$ by $g_{0} \equiv 1 / \sqrt{M}=g / \sqrt{M_{s}}$. The $4+1$ Lagrangian takes the form:

$$
\mathcal{L}_{5}=-\frac{1}{4} \operatorname{Tr}\left(\hat{F}_{M N} \hat{F}^{M N}\right), \quad \hat{F}_{M N}^{a}=\partial_{M} \hat{A}_{N}^{a}-\partial_{N} \hat{A}_{M}^{a}+g_{0} f^{a b c} \hat{A}_{M}^{b} \hat{A}_{N}^{c}
$$

where $a$ is the gauge index and $f^{a b c}$ is the structure constant.

(ii) Momentum Space Expansion and Truncation

A necessary gauge-covariant boundary condition is:

$$
F^{5 N}=F^{N 5}=0, \quad \text { at } x^{5}=R_{I, I I}
$$

This removes unwanted gauge invariant vector field strengths that transform as a 4 -vector in the $3+1$ theory. The simplest gauge choice realizing these boundary conditions is to impose Neumann conditions for $\hat{A}_{\mu}$ with $\mu=0,1,2,3$, i.e. $\partial \hat{A}_{\mu} / \partial x^{5}=0$, at $x_{5}=R_{I, I I}$, and Dirichlet conditions for the $3+1$ "scalars" $\hat{A}_{5}$, i.e. $\hat{A}_{5}=0$ at $x_{5}=R_{I, I I}$. The lowest energy physical $\hat{A}_{\mu}$ modes are massless, independent of $x_{5}$, and form the usual $3+1$ gauge field. We can further choose an axial gauge $\chi^{A} \hat{A}_{A}=0$ where $\chi^{A}$ is a 5 -vector normal to the branes. This sets $\hat{A}^{5}=0$. We will adopt this gauge choice after the momentum space expansion.

\footnotetext{
${ }^{2}$ The ordinary spacetime coordinates are labeled by $x^{\mu}, \mu=0,1,2,3$, and the fifth dimension by $x^{5}$ to avoid confusion with $x^{4}=i c t$; Capital letters denote the bulk coordinates, $M, N=0,1,2,3,5$.
} 
We thus can expand the 4 -vector potential $\hat{A}_{\mu}\left(x_{\mu}, x_{5}\right)$ in a Fourier cosine series,

$$
\hat{A}_{\mu}=\frac{1}{\sqrt{R}}\left[A_{\mu}^{0}+\sqrt{2} \sum_{n=1}^{+\infty} A_{\mu}^{n}\left(x_{\mu}\right) \cos (n \theta)\right], \quad \theta=\frac{\pi x_{5}}{R}
$$

where we have suppressed the gauge index $a$ and $A^{0}$ is the $n=1$ zero-mode. The fifth component $\hat{A}_{5}\left(x_{\mu}, x_{5}\right)$ is given by a Fourier sine series,

$$
\hat{A}_{5}=\sqrt{\frac{2}{R}} \sum_{n=1}^{+\infty} A_{5}^{n}\left(x_{\mu}\right) \sin (n \theta) .
$$

and this has no zero-mode. The coefficients of the expansions are:

$$
\begin{array}{ll}
A_{\mu}^{0}=\frac{1}{2 \sqrt{R}} \int_{0}^{R} d x_{5} \hat{A}_{M}\left(x_{\mu}, x_{5}\right) ; \\
A_{\mu}^{n}=\frac{1}{\sqrt{2 R}} \int_{0}^{R} d x_{5} \hat{A}_{M}\left(x_{\mu}, x_{5}\right) \cos (n \theta) ; \quad n=1, \cdots,+\infty \\
A_{5}^{n}=\frac{1}{\sqrt{2 R}} \int_{0}^{R} d x_{5} \hat{A}_{5}\left(x_{\mu}, x_{5}\right) \sin (n \theta) ; \quad n=1, \cdots, \infty
\end{array}
$$

The non-hat vector field $A_{M}^{n}$ has mass dimension +1 .

The field strengths read,

$$
\begin{aligned}
\hat{F}_{\mu \nu}\left(x_{\alpha}, x_{5}\right)= & \frac{1}{\sqrt{R}}\left\{\left[\partial_{[\mu} A_{\nu]}^{0}+\sum_{n=1}^{+\infty} \cos (n \theta) \partial_{[\mu} A_{\nu]}^{n}\right]\right. \\
& \left.+\frac{g}{\sqrt{M_{s} R}} f^{a b c}\left[A_{\mu}^{0}+\sqrt{2} \sum_{n=1}^{+\infty} A_{\mu}^{n} \cos (n \theta)\right]\left[A_{\mu}^{0}+\sqrt{2} \sum_{m=1}^{+\infty} A_{\mu}^{m} \cos (m \theta)\right]\right\}
\end{aligned}
$$

the color indices on the vector fields are supressed in this equation as well as in the following equations. Integrating over $x^{5}$ we obtain the effective $3+1$ theory.

If we now impose the axial gauge $A_{5}\left(x_{\mu}, x_{5}\right) \equiv 0$, the effective Lagrangian after integrating over $x_{5}$ and truncating at the $N$ th KK mode takes the form:

$$
\begin{aligned}
\mathcal{L}_{4}= & \left(\partial_{\mu} A_{\nu}^{0}-\partial_{\nu} A_{\mu}^{0}+\frac{g}{\sqrt{M_{s} R}} f^{a b c} A_{\mu}^{0} A_{\nu}^{0}\right)^{2}+\sum_{n=1}^{N}\left(\partial_{\mu} A_{\nu}^{n}-\partial_{\nu} A_{\mu}^{n}\right)^{2} \\
& +\frac{2 g}{\sqrt{M_{s} R}} f^{a b c} \sum_{n=1}^{N}\left[\partial_{[\mu} A_{\nu]}^{0} A^{n \mu} A^{n \nu}+\partial_{[\mu} A_{\nu]}^{n}\left(A^{0 \mu} A^{n \nu}+A^{n \mu} A^{0 \nu}\right)\right] \\
& +\frac{g}{\sqrt{2 M_{s} R}} f^{a b c} \sum_{n, m, l=1}^{N} \partial_{[\mu} A_{\nu]}^{n} A^{m \mu} A^{l \nu} \Delta_{1}(n, m, l)
\end{aligned}
$$




$$
\begin{aligned}
& +\frac{g^{2}}{M_{s} R} f^{a b c} f^{a d e} \sum_{n=1}^{N}\left(A_{\mu}^{0} A_{\nu}^{0} A^{n \mu} A^{n \nu}+\text { all permutations }\right) \\
& +\frac{g^{2}}{2 M_{s} R} f^{a b c} f^{a d e} \sum_{n, m, l, k=1}^{N} A_{\mu}^{n} A_{\nu}^{m} A^{l \mu} A^{k \nu} \Delta_{2}(n, m, l, k) \\
& +\sum_{n=1}^{N}\left(\frac{n \pi}{R}\right)^{2} A_{\mu}^{n} A^{n \mu}
\end{aligned}
$$

where the $\Delta_{i}$ are defined as:

$$
\begin{aligned}
\Delta_{1}= & \delta(n+m-l)+\delta(n-m+l)+\delta(n-m-l) \\
\Delta_{2}= & \delta(n+m-l-k)+\delta(n+m+l-k)+\delta(n+m-l+k) \\
& +\delta(n-m+l+k)+\delta(n-m-l-k)+\delta(n-m+l-k)+\delta(n-m-l+k) .
\end{aligned}
$$

The zero mode has the canonical $3+1$ kinetic term with field strength:

$$
F_{\mu \nu}^{0 a}=\partial_{\mu} A_{\nu}^{0 a}-\partial_{\nu} A_{\mu}^{0 a}+\tilde{g} f^{a b c} A_{\mu}^{0 b} A_{\nu}^{0 c},
$$

Hence, $\tilde{g} \equiv g / \sqrt{M_{s} R}$ is the dimensionless low-energy $3+1$ coupling constant. If the truncation $N=M_{s} R$ on the number of the KK modes is introduced then $\tilde{g} \equiv g / \sqrt{N}$. A perturbative theory of the zero mode requires $\tilde{g}<\mathcal{O}(1)$, i.e., $g<\sqrt{M_{s} R}$ or $M>1 / R$.

\section{(iii) Comparison to Aliphatic Theory}

Now, consider again the aliphatic theory with the gauge structure $S U(3)_{0} \times S U(3)_{1} \times$ $\ldots \times S U(3)_{N}$, where the vector potentials are $A_{\nu}^{j} a$. In addition, there are a set of $\Phi_{i}$ fields which straddle the $i$ th and $i+1$ th $S U(3)$ gauge groups. The Lagrangian takes the form as in eqn.(1.1), and the mass spectrum as in eqn.(1.3). The gauge fields $A_{\mu}^{j}$ can be expressed as linear combinations of the mass eigenstates $\tilde{A}_{\mu}^{n}$ as:

$$
A_{\mu}^{j}=\sum_{n=0}^{N} a_{j n} \tilde{A}_{\mu}^{n}
$$

The $a_{n j}$ form a normalized eigenvector $\left(\vec{a}_{n}\right)$ associated with the $n$th $n \neq 0$ eigenvalue and has the following components:

$$
a_{n j}=\sqrt{\frac{2}{N+1}} \cos \left(\frac{2 j+1}{2} \gamma_{n}\right), \quad j=0,1, \ldots, N,
$$

The eigenvector for the zero-mode, $n=0$, is always $\vec{a}_{0}=\frac{1}{\sqrt{N+1}}(1,1, \ldots, 1)$. The orthogonality between the eigenvectors is due to:

$$
\sum_{j=0}^{N} \cos \left(\frac{2 j+1}{2} \gamma_{n}\right) \cos \left(\frac{2 j+1}{2} \gamma_{m}\right)=\delta(n-m) \frac{N+1}{2}, \quad n, m \neq 0 \ll N
$$


with $\gamma_{n}=\frac{n \pi}{N+1}$. We can now rewrite the Lagrangian eqn.(1.1) in the mass eigenstates of the vector bosons $\left(\tilde{A}_{\mu}^{n}\right)$ and derive the interactions between them.

Let us now compare the KK reduction of the five-dimensional theory, eqn.(3.16), and the aliphatic $\left(S U(3)^{N+1}, \Phi^{N}\right)$ theory at the level of interactions. In the aliphatic theory, as far as the mass spectrum is concerned, there are three free parameters, namely, the gauge coupling constant $g_{L}$, the total number of $S U(3)$ groups $N+1$ and the VEV of the Higgs field $v$. As we discussed earlier, one can arrange the parameters of the $S U(3)^{N+1}$ theory to fix the ratio $g_{L} v / \sqrt{2}(N+1)=\frac{1}{R}$, such that the spacing of the linear mass spectrum at $n<<N$ is completely determined and the mass spectrum of the two theories matches.

To compare the Lagrangian's couplings we substitute eqn.(3.18) into the gauge part of the Lagrangian eq.(1.2):

$$
\mathcal{L}_{\text {gauge }}=-\frac{1}{4} \sum_{j=0}^{N}\left(\sum_{n=0}^{N} a_{j n} \partial_{[\mu} A_{\nu]}^{n}+g_{L} f^{a b c} \sum_{n=0}^{N} \sum_{m=0}^{N} a_{j n} a_{j m} A_{\mu}^{n} A_{\nu}^{m}\right)^{2}
$$

Isolating the zero-mode, $\tilde{A}_{\mu}^{0}$, and, using orthonormality, we can write down the canonical kinetic terms:

$$
\mathcal{L}_{g, k i n}=-\frac{1}{2}\left(\partial_{[\mu} \tilde{A}_{\nu]}^{0}+\frac{g_{L}}{\sqrt{N+1}} f^{a b c} \tilde{A}_{\mu}^{0} \tilde{A}_{\nu}^{0}\right)^{2}+\sum_{n=1}^{N}\left(\partial_{[\mu} \tilde{A}_{\nu]}^{n}\right)^{2} .
$$

The trilinear gauge coupling takes the form:

$$
\mathcal{L}_{g, 3 A}=-\frac{1}{4} \sum_{n, m, l \neq(0,0,0)}\left(\sum_{j=0}^{N} a_{j n} a_{j m} a_{j l}\right) g_{L} f^{a b c} \partial_{[\mu} \tilde{A}_{\nu]}^{n} \tilde{A}^{m \mu} \tilde{A}^{l \nu} .
$$

Using pairwise summations and orthogonality:

$$
\sum_{j=0}^{N} a_{j n} a_{j m} a_{j l}=\left\{\begin{array}{cc}
\sqrt{\frac{1}{N+1}}[\delta(n) \delta(m-l)+\delta(m) \delta(n-l)+\delta(l) \delta(n-m)], & \\
\sqrt{\frac{1}{2(N+1)}} \Delta_{1}(n, m, l), & n, m, l \neq 0 ;
\end{array}\right.
$$

where $\Delta_{1}$ is defined previously. Similarly, the quadrilinear couplings take the form:

$$
\mathcal{L}_{g, 4 A}=-\frac{1}{4} \sum_{n, m, l, k \neq(0,0,0)}\left(\sum_{j=0}^{N} a_{j n} a_{j m} a_{j l} a_{j k}\right) g_{L} f^{a b c} g_{L} f^{a d e} \tilde{A}_{\mu}^{n} \tilde{A}_{\nu}^{m} \tilde{A}^{l \mu} \tilde{A}^{k \nu},
$$

with the coefficients,

$$
\sum_{j=0}^{N} a_{j n} a_{j m} a_{j l} a_{j k}=\left\{\begin{array}{cc}
\frac{1}{N+1}, & \text { two of }(\mathrm{n}, \mathrm{m}, \mathrm{l}, \mathrm{k}) \\
\frac{1}{2(N+1)} \Delta_{2}(n, m, l, k), & \text { are zero, remainders are equal; }
\end{array}\right.
$$


We see that $\Delta_{2}(n, m, l, k)$ is exactly the same function defined in the discussion of truncated momentum space expansion. Thus, we see that, defining the gauge coupling constant $\bar{g}=g_{L} / \sqrt{N+1}$ of the unbroken $S U(3)$ in the aliphatic theory to satisfy $\bar{g}=\tilde{g}=$ $g / \sqrt{M_{s} R}$, the couplings and Feynman rules in the two theories agree perfectly. This completes the demonstration of the equivalence.

In both theories, there are three fundamental parameters, i.e., $M_{s}, M=1 / g_{0}^{2}, R$ in the KK reduced theory and $g_{L}, N, v$ in the aliphatic theory. The mappings between them are $N+1=M_{s} R, g_{L}=\sqrt{M_{s} / M}$ and $v=\sqrt{M_{s} M}$, and they are valid up to the scale $v$. Measurement of the zero mode interactions give us $\bar{g}=\tilde{g}$. The mass of the first KK mode tells us $\frac{g_{L} v}{N+1}=\frac{1}{R}$. Hence, two of the three parameters can be determined, leaving $M_{s}=g_{L} v$ undetermined in the two theories. The mass of $M_{2}$ will test the linear spacing between the KK modes, rather than give further constraints on the parameters.

Suppose we had a bulk $5+1$ theory. Then we would have a different structure for the low energy effective theory, and we would have a correspondingly different lattice theory. No longer would the theory be an aliphatic model, and would appear then as a more complex closed structure, first an aromatic hydrocarbon, eventually a polymerized molecular solid state. One can generalize our construction to theories in two extra-dimensions with size $R_{1} \times R_{2}$. The low energy effective theory would be different.

The simplest case is the limit of a single plaquette in the two compact dimensions of $5+1$, the analogue of an Eguchi-Kawai model [7]. The low energy theory would contain the gluon zero-mode, which is the rotational zero-mode of such a configuration, and a doubly degenerate pair of colorons as the first KK modes, and a third heavy singlet. One can expand the single plaquette construction to multi-plaquette construction, which requires $(N+1) \times(M+1) S U(4)$ and $2 N \times M+N+M \Phi_{i}$ fields, where $N=R_{1} / a_{1}$ and $M=R_{2} / a_{2}$ and $a_{1}, a_{2}$ are spacings between the 3 -branes.

It is interesting that ultimately the lattice structure must also reflect the homotopy of the extra dimensions. If there is a "hole" in the space of the extra dimensions, there must be corresponding nontrivial paths through the Higgs field links that match the non-contractable loops in that space.

\section{Incorporation of Fermions}

The models we presented for the gauge bosons in the bulk can easily accommodate fermions and bosons in the bulk. 


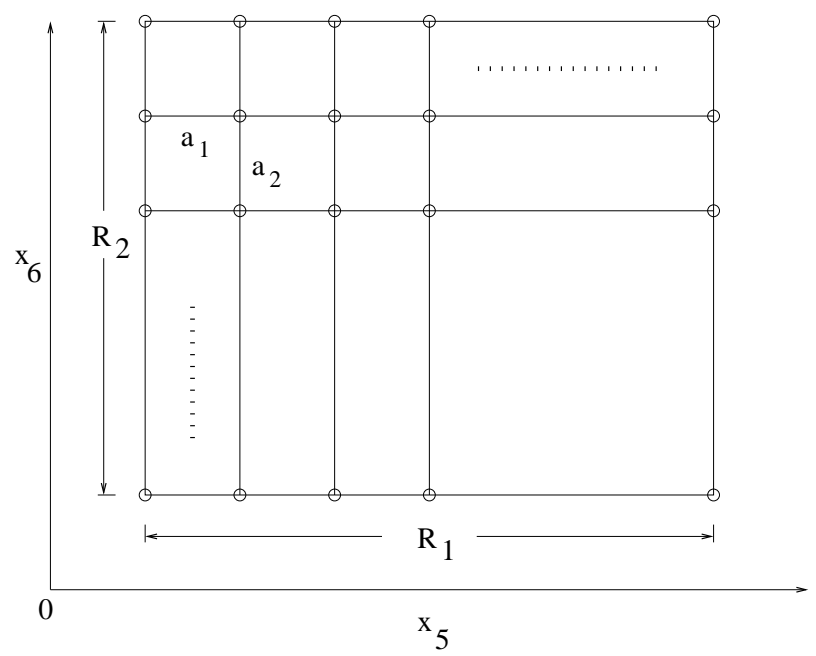

Figure 2: The geometric interpretation for the plaquette model with two extra dimensions. The Eguchi-Kawai model corresponds to a single plaquette. At each circle, there is a 3brane with one $S U(3)$ symmetry.

The Lagrangian for a fermion in the five dimensional bulk which is charged under the bulk $S U(3)$ symmetry is given by

$$
\mathcal{L}_{5}\left(x^{\mu}, x^{5}\right)=\bar{\Psi}\left(i \gamma^{\mu} D_{\mu}-\gamma_{5} D_{5}\right) \Psi-\frac{1}{4} \operatorname{Tr}\left(F^{M N} F_{M N}\right),
$$

where the covariant derivative is defined previously. The five dimensional fermion is non-chiral, hence its zero mode upon the compactification of the 5 th dimension can be non-chiral, unless the Lorentz group in five dimensions $S O(4,1)$ is explicitly broken by imposing different boundary conditions for the left-handed component, $\Psi_{L}$, and the righthanded component, $\Psi_{R}$. The boundary conditions also prevents $\Psi$ from having a bare mass term in the bulk. Consider, for example, the following boundary condition,

$$
\left.\frac{\partial}{\partial x_{5}} \Psi_{L}\right|_{x_{5}=0, R}=0 ;\left.\quad \Psi_{R}\right|_{x_{5}=0, R}=0 .
$$

The Neumann boundary condition for $\Psi_{L}$ ensures that there is a massless left-handed four dimensional fermion on the brane, while the Dirichlet conditions makes all the righthanded modes massive. Upon compactification, $\Psi_{L}$ can be decomposed into a cosine series and $\Psi_{R}$ can be decomposed into a sine series. The masses of the fermion KK modes are given by $M_{L / R, n}=n \pi / R$.

In the aliphatic model, consider $N+1$ fermions $\Psi_{n}(n=0 \cdots N)$, each of which is charged under the corresponding $S U(3)_{n}$ symmetry. The Higgs fields $\Phi_{n}$ which is $(\overline{3}, 3)$ 
under the two neighboring $S U(3)$ symmetries provides the nearest neighbor couplings between the fermion fields. The effective Lagrangian takes the form

$\mathcal{L}_{\text {fermion }}\left(x^{\mu}\right)=\sum_{n=0}^{N} \bar{\Psi}_{n, L / R} \not \supset \Psi_{n, L / R}+M_{f}\left[\overline{\Psi_{n, L}}\left(\frac{\Phi_{n+1}^{\dagger}}{v} \Psi_{n+1, R}-\Psi_{n, R}\right)-\overline{\Psi_{n, R}}\left(\Psi_{n, L}-\frac{\Phi_{n}}{v} \Psi_{n-1, L}\right)\right]$,

where $\not D$ is defined as the four dimensional covariant derivative.

In the aliphatic model, the boundary conditions in eqn. (4.28) can be translated into $\Psi_{0, R}=\Psi_{N, R}=0$ and $\Psi_{L, N}-\Psi_{L, N-1}=0$. As a result, in the vacuum where $\Phi_{n}$ has non-zero VEV $v$, the mixed mass terms for the left-handed and right-handed fermions are

$$
\begin{aligned}
\mathcal{L}_{\text {mass }} & =M_{f}\left\{\overline{\Psi_{0, L}} \Psi_{1, R}+\sum_{n=1}^{N-1}\left[\overline{\Psi_{n, L}}\left(\Psi_{n+1, R}-\Psi_{n, R}\right)-\overline{\Psi_{n, R}}\left(\Psi_{n, L}-\Psi_{n-1, L}\right)\right]\right\} \\
& = \\
& \left(\overline{\Psi_{0, L}}, \cdots, \overline{\Psi_{N-1, L}}\right) M\left(\Psi_{1, R}, \cdots, \Psi_{N-1, R}\right)^{T}
\end{aligned}
$$

where the $N \times(N-1)$ mass matrix $M$ takes the form

$$
M=M_{f}\left(\begin{array}{cccc}
1 & 0 & \cdots & 0 \\
-1 & 1 & \ldots & 0 \\
& & \ldots & \\
0 & \cdots & -1 & 1 \\
0 & \ldots & 0 & -1
\end{array}\right) .
$$

To calculate the mass eigenvalues and eigenstates for the right-handed components, one can diagonalize the $(N-1) \times(N-1)$ matrix $M^{\dagger} M$,

$$
M^{\dagger} M=\left|M_{f}\right|^{2}\left(\begin{array}{ccccc}
2 & -1 & 0 & \cdots & 0 \\
-1 & 2 & -1 & \cdots & 0 \\
0 & -1 & 2 & \cdots & 0 \\
& & & \cdots & \\
0 & 0 & \cdots & -1 & 2
\end{array}\right) .
$$

Therefore, the eigenvalues of the right-handed fermions are

$$
M_{R, n}=2 M_{f} \sin \left(\frac{n \pi}{2 N}\right), \quad n=1,2, \cdots, N-1 .
$$

In terms of the mass eigenstates $\tilde{\Psi}_{n, R}$,

$$
\Psi_{n, R}=\sqrt{\frac{N}{2}} \sum_{k=1}^{N-1} \sin \left(n \frac{k \pi}{N}\right) \tilde{\Psi}_{k, R} .
$$


The mass eigenvalues of the left-handed fermions can be calculated from the $N \times N$ matrix $M M^{\dagger}$, which takes the following form,

$$
M M^{\dagger}=\left|M_{f}\right|^{2}\left(\begin{array}{ccccc}
1 & -1 & 0 & \cdots & 0 \\
-1 & 2 & -1 & \cdots & 0 \\
0 & -1 & 2 & \cdots & 0 \\
& & & \cdots & \\
0 & 0 & \cdots & -1 & 1
\end{array}\right) .
$$

Hence, the eigenvalues of the left-handed fermions are similar to those of the gauge bosons,

$$
M_{n, L}=2 M_{f} \sin \left(\frac{n \pi}{2 N}\right), \quad n=0 \cdots N-1 .
$$

Hence, the left-handed fermions have a massless zero mode. The massive modes have the same mass as those of the right-handed fermions, thus form massive vector pairs.

The eigenvectors of the left-handed fermions also have the same structure as that of the gauge bosons, namely, in terms of the mass eigenstates $\tilde{\Psi}_{k, L}$,

$$
\Psi_{n, L}=\sqrt{\frac{N}{2}} \sum_{k=0}^{N-1} \cos \left(\frac{2 n+1}{2} \frac{k \pi}{N}\right) \tilde{\Psi}_{k, L} .
$$

Note that left-handed fermions have a cos expansion, while the right-handed fermions assume a sin expansion.

In the limit that $n \ll N$, a linear massive spectrum is recovered for both right-handed and left-handed fermions, in which $M_{n}=M_{f} \frac{n \pi}{N}$. Since the masses of the KK modes for a $D=5$ fermion are $M_{L / R, n}=\frac{n \pi}{R}$, one reproduces the linear spectrum for the KK theory by choosing $M_{f}=\frac{N}{R}$.

The coupling between the fermions and the gauge field takes the following form in their mass eigenstate basis,

$$
\begin{gathered}
\mathcal{L}_{f f A}=\sum_{n, m, l \neq(0,0,0)} g_{L} \tilde{\bar{\Psi}}_{n, L} \gamma^{\mu} \tilde{A}_{\mu m} \tilde{\Psi}_{l, L} \Delta_{n, m, l}+g_{L} \tilde{\bar{\Psi}}_{0, L} \gamma^{\mu} \tilde{A}_{\mu 0} \tilde{\Psi}_{0, L} \\
+\sum_{n, m, l \neq 0, N} g_{L} \tilde{\bar{\Psi}}_{n, R} \gamma^{\mu} \tilde{A}_{\mu m} \tilde{\Psi}_{l, R} \Delta_{1}(n, m, l),
\end{gathered}
$$

in which $\Delta_{1}$ is defined as the sum in eqn.(3.24).

One can also write down the effective Lagrangian for a massless complex boson in the bulk in our frame work. Consider $N+14 \mathrm{D}$ complex scalar with the following Lagrangian,

$$
\mathcal{L}_{\text {boson }}=\sum_{i=0}^{N}\left|D_{\mu} \phi_{i}\right|^{2}-M_{b}^{2} \sum_{i=1}^{N}\left|\phi_{i-1}-\frac{1}{v} \Phi_{i} \phi_{i}\right|^{2} .
$$


In the vacuum in which $\left\langle\Phi_{i}\right\rangle=v$, the scalars have the mass terms $-M_{b}^{2} \sum_{i=1}^{N}\left|\phi_{i-1}-\phi_{i}\right|^{2}$. It can diagonalized by

$$
\phi_{j}=\frac{1}{N+1} \sum_{n=1}^{N} e^{i 2 \pi n j /(N+1)} \tilde{\phi}_{n},
$$

with the mass spectrum

$$
M_{n, b}=2 M_{b} \sin \gamma_{n}, \quad n=0,1, \cdots N
$$

Each level with $n \neq 1$ is degenerate with the level $N-n$, while the zero mode is a singlet. This doubling of energy levels corresponds to the mode expansion in $x^{5}$ in terms of 1 , $\sin \left(n \pi x^{5} / R\right)$ and $\cos \left(n \pi x^{5} / R\right)$, where the sine and cosine terms are degenerate modes.

\section{Renormalization of gauge coupling constant}

Unlike the compactified continuum theory, the spontaneously broken gauge theory $\left(S U(3)^{N+1}\right.$ , $\Phi^{N}$ ) is a renormalizable field theory. Thus, we can discuss the scale dependence of the coupling strength $\bar{g}(\mu)$ of the unbroken $S U(3)$ via the radiative corrections. The standard method of constructing effective field theories at each stage of the decoupling of the massive modes is at best confusing. One problem is that when decoupling the $n_{t h} \mathrm{KK}$ mode with mass $M_{n}$, the decoupling methods tells us to construct an effective theory with one zero mode and $n-1 \mathrm{KK}$ modes which should be taken to be massless at the decoupling scale $M_{n}$, this is, the effective field theory will have a gauge symmetry $S U(3)^{n}$. But the original theory tells us that all $S U(3)^{N+1}$ is broken to $S U(3)$ at the scale $v$, and it is different from breaking the $S U(3)$ symmetries one by one at each $M_{n}$. Another problem is that, at two or higher loop level, one necessarily encounters loops with both light and heavy KK modes, such that it is confusing to even define a proper decoupling scale.

However, one can define the effective coupling constant $\bar{g}\left(\mu^{2}\right)$ in the momentum subtraction scheme [8], e.g., as the triple gluon (zero mode) vertex. All the external legs have the momentum $q^{2}=-\mu^{2}$. The effective coupling $\bar{g}\left(\mu^{2}\right)$ is governed by the equation

$$
\frac{\partial \bar{g}\left(\mu^{2}\right)}{\partial \ln \mu^{2}}=\beta\left(\bar{g}\left(\mu^{2}\right)\right),
$$

and its evolution can be calculated in any order of perturbation in the full spontaneously broken $\left(S U(3)^{N+1}, \Phi^{N}\right)$ theory, including all KK modes. Strictly speaking, one gets a set of coupled differential equations, since the $\beta$ function in eqn. (5.42) depends on the triple vector boson couplings $\bar{g}_{0 n n}\left(\mu^{2}\right)$, each running according to its own evolution 
equation. The problem radically simplifies at 1-loop level and in the approximation [8] where one assumes that the KK modes that appears in the loops satisfy $-\mu^{2} \leq\left(M_{i}^{2}+M_{j}^{2}\right)$. Moreover, in 1-loop calculation one can use the relationship between tree level couplings, namely, $\bar{g}=\bar{g}_{0 n n}$ for any $n$.

Thus, at 1-loop level, the running of the gauge coupling constant $\bar{g}$ between the scales $\left(M_{n}, M_{n-1}\right)$ involves the $n$ modes which are lighter then $M_{n}$, as a result the running can be described by,

$$
\frac{d \bar{g}}{d \log \mu}=-\left[n \frac{\beta}{4 \pi^{2}}\right] \bar{g}^{3}, \quad M_{n-1} \leq \mu \leq M_{n}
$$

in which $\beta$ is the 1-loop RGE coefficient of a pure $S U(3)$ theory. Hence, given the measured coupling constant $\alpha\left(M_{Z}\right)$ at low energy, the gauge coupling constant at energy scale $\mu$ is given by

$$
\alpha^{-1}(\mu)=\alpha^{-1}\left(M_{Z}\right)-\frac{\beta}{4 \pi}\left[\ln \left(\frac{M_{1}}{M_{Z}}\right)+\sum_{n=2}^{n_{\max }} n \ln \left(\frac{M_{n}}{M_{n-1}}\right)+\left(n_{\max }+1\right) \ln \left(\frac{\mu}{M_{n_{\max }}}\right)\right],
$$

where $M_{n_{\max }} \leq \mu<M_{n_{\max }+1}$. One can sum up the series to arrive at,

$$
\alpha^{-1}(\mu)=\alpha^{-1}\left(M_{Z}\right)-\frac{\beta}{4 \pi} \ln \left(\frac{\mu}{M_{Z}}\right)-\frac{\beta}{4 \pi} n_{\max } \ln \left(\frac{\mu}{M_{1}}\right)+\frac{\beta}{4 \pi} F\left(M_{n}\right)
$$

in which the factor $F \equiv \ln \left(\frac{\Pi_{n=1}^{n \max } M_{n}}{M_{1}^{n m a x}}\right)$ depends on what kind of the KK spectrum we work with. The linear spaced KK spectrum from the dimensionally reduced continuum theory gives:

$$
F_{\text {lin }}=\ln \left(n_{\max } !\right)
$$

while the spectrum from the aliphatic model as in eqn. (2.5) gives:

$$
F_{a l i}=\ln \left(\frac{\prod_{n=1}^{n_{\max }} \sin \left(\frac{n \pi}{2(N+1)}\right)}{\sin \left(\frac{\pi}{2(N+1)}\right)^{n_{\max }}}\right) .
$$

Eqn. (5.45) with $F_{\text {lin }}$ is derived in [5] and [6], it shows a power law behavior of the gauge coupling constant. The differences between $F_{l i n}$ and $F_{\text {ali }}$ provides an interesting measure on how much the aliphatic mode deviates from the continuum theory at a quantum level. In Fig. (3), we plot $F_{l i n}$ and $F_{\text {ali }}$ as a function of $n_{\max }$, keeping $N$ fixed. Fig. (4) shows $F_{\text {lin }}$ and $F_{\text {ali }}$ as a function of $N$, while $n_{\max }$ is fixed.

It can be seen from the figures that when $n_{\max }$ is small compared to $N$, the two theories agree very well in their $\beta$ functions, since in this region, the aliphatic model gives an excellent approximation of the linear spectrum from the compactified continuum 




Figure 3: $F_{\text {lin }}$ (solid line) and $F_{\text {ali }}$ (dashed line) as functions of $n_{\max } . N=20$ is chosen for the plot.

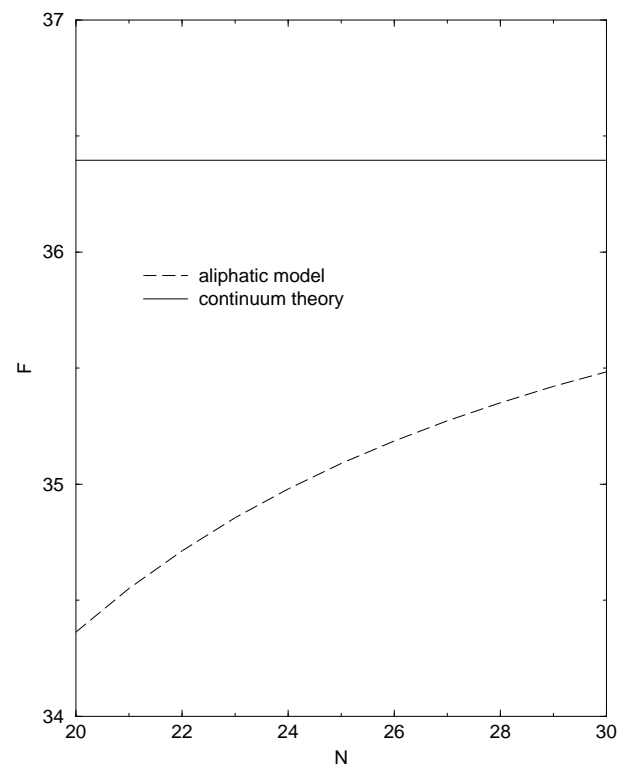

Figure 4: $F_{\text {lin }}$ (solid line) and $F_{\text {ali }}$ (dashed line) as functions of $N \cdot n_{\max }=18$ is chosen for the plot. 
theory, as we expected. However, even at $n_{\max }$ close to $N$, the deviation of $F_{\text {ali }}$ from $F_{\text {lin }}$ is less than $10 \%$.

We don't expect things will be drastically different at two or higher loop levels. This observation suggests to us that the aliphatic model provides a good approximation to the continuum theory even at an energy scale close to $v$, the "error" in approximating the continuum theory lies in the finite size of the lattice, i.e., the separation between the two nearest branes. One can always reduce the "error" by adding more branes, thus increasing $N$ and reducing the inter-brane separation. It also suggests to us that, if one wants to modify the aliphatic theory such that it will produce exactly the linear spectrum up to $M_{N}$, one only needs to add higher order operators, perhaps the type of operators which mimic the couplings between the next-to-nearest branes.

\section{Discussion and Conclusion}

In eq.(2.3) we assume that one can lift the Higgs mass to a high energy scale above the cut-off scale $M_{s}$. The Higgs degrees of freedom then decouple from the theory, and only the Nambu-Goldstone modes remain, which are eaten by the KK modes to give mass. This is a large coupling limit of the Higgs theory in which the VEV is held fixed, i.e., $v \sim M / \sqrt{\lambda}$ where $M \rightarrow \infty$ and $\lambda \rightarrow \infty$ together. However, such a theory violates perturbative unitarity. On the other hand, the effective low energy theory is a gauged chiral Lagrangian with $f_{\pi} \sim v$. This theory is a pertubatively sensible one (and is renormalizeable as expansion in $1 / v^{p}$ ) in the low energy limit, however, the perturbative unitarity breakdown occurs when $\sqrt{s} \gtrsim v$. Essentially, longitudinal KK mode scattering must violate perturbative unitarity when $s \gtrsim 4 \pi v^{2}$. This is the Lee-Quigg-Thacker bound which applies to, e.g., electroweak symmetry breaking for $W W$ scattering [3].

We see, from eq.(2.7), that this failure of unitarity corresponds to energy scales approaching $s \gtrsim 4 \pi N^{2} / g_{L}^{2} R^{2} \sim 4 \pi N^{2} M / R^{2} M_{s}$. As we have seen, our theory corresponds to a $4+1$ theory with a dimensional coupling given by $g_{0}$. We would generally expect this theory to violate perturbative unitarity for $s \gtrsim 4 \pi M_{s} / g_{0}^{2}$, hence, by comparison that indeed $s \gtrsim 4 \pi N^{2} M / R^{2} M_{s} \sim 4 \pi M_{s} / g_{0}^{2}$. Hence the perturbative unitarity violation inherent in the large coupling constant of the parent $D=5$ theory is matched by the unitarity breakdown in the effective $3+1$ theory.

The separation of scales, $N \sim M_{s} / M_{c}>>1$ is a requirement of very low mass, or infrared states, in an essentially strong-dynamical theory at the scale $M_{s}$, In all cases in 
nature where this phenomenon occurs and is understood, there is an attendant custodial symmetry. The theory we have presented in $3+1$ dimensions imitates arbitrarily well a $4+1$ theory, and this dynamical issue does not seem to arise. The infrared physics scale, the "effective compactification scale," is $M_{c} \sim M_{s} / N$ and apparently occurs accidentally because $N$, the number of independent gauge groups in the contruction, is very large.

One might have thought that the separation of the compactification scale and the fundamental scale in extra-dimensional models would involve, at least accidentally, approximate classical scale invariance (this is the custodial symmetry in QCD of, e.g., the ratio $\Lambda_{Q C D} / M_{\text {Planck }}$ in the sense that "classical scale invariance" corresponds to setting the $\beta$-function of QCD to zero). The QCD coupling in our theory turns out to be suppressed as $\alpha_{Q C D} \sim M_{s} \alpha_{0} / N$, where $\alpha_{0}=g_{0}^{2} / 4 \pi$ is the dimensional $4+1$ gauge coupling. To take $N$ arbitrarily large thus implies that the theory must have a slowly running dimensionless couplng constant (remniscent of "walking technicolor") in $D=4$ on scales well below $M_{s}$, so it does appear that quantum scale breaking effects are under control, and it seem that classical scale invariance is acting as the custodial symmetry afterall. However, the trace of the stress-tensor in $D=5$ is nonzero classically, and the theory has explicit scale breaking, owing to the $D=5$ dimensional coupling constant. The nonzero trace, $T_{\mu}^{\mu} \propto G_{\mu \nu}^{a} G^{a \mu \nu}$ in $D=5$ must match onto the KK masses as in $D=4$, since the KK masses are seen as explicit sources of scale breaking on all scale from $M_{c}$ to $M_{s}$. It is therefore quite puzzling as to what, if anything, we may we invoke as the custodial symmetry of the scale hierarchy in extra dimensions when $N$ is large. Is this a counter example to the requirememnt of having an explicit custodial symmetry, an artifact of large $N$ ?

In conclusion, We have constructed a manifestly gauge invariant description of $n$ KK modes for an $S U(m)$ gauge theory in the bulk. We showed in this paper the fourdimensional KK theory deducted from a compactified five-dimensional $S U(3)$ theory can be considered as a $\left(S U(3)^{N+1}, \Phi^{N}\right)$ theory, in which the $S U(3)^{N+1}$ gauge symmetry is spontaneously broken to $S U(3)$. This theory owes its structure to a transverse lattice theory with one extra dimension. The three dimensional parameters of the original KK theory, the string (cut-off) scale $M_{s}$, the compactification radius $R$ and the fivedimensional gauge coupling $g_{0} \equiv \sqrt{M^{-1}}$, determine the structure of the $\left(S U(3)^{N+1}, \Phi^{N}\right)$ theory: $N=M_{s} R$, the coupling constant of the unbroken $S U(3) \bar{g}=1 / \sqrt{M R}$, and the scale $v=\sqrt{M_{s} M}$ of the spontaneous symmetry breaking $S U(3)^{N+1} \rightarrow S U(3)$.

The approach maintains manifest gauge invariance. Is it possible to construct anal- 
ogous effective Lagrangians which maintain SUSY and general covariance for yielding KK modes of gravity? And how are the topological aspects of extra dimensional gauge theories [9] expressed in an effective Lagrangian such as this?

(Note added:) Upon completion of this work the preprint of Arkani-Hamed, Cohen and Georgi, [11], appeared which uses a technicolor-like condensate in place of our explicit Higgs fields, $\Phi_{n}$, but obtains essentially the identical construction as a chiral Lagrangian. Georgi's moose notation, used in [11], may be a useful way to extend to higher dimensions such as $5+1$ with 2 compact dimensions, whence the theory may be graphically represented as a "moose lattice," and the anomaly free incorporation of fermions is automatic.

\section{Acknowledgements}

We wish to thank W. Bardeen, Hsin Chia Cheng and Martin Schmaltz for useful discussions. One of us (SP) wishes to thank P. Chankowski and M. Misiak for useful discussions and acknowledge the kind hospitality of the Fermilab theory group where this work originated. Research by $\mathrm{CH}$ and JW was supported by the U.S. Department of Energy Grant DE-AC02-76CHO3000. Research by SP was supported by the Polish State Committee for Scientific Research, grant KBN 2 P03B 06018 (2000-01). 


\section{References}

[1] I. Antoniadis, Phys.Lett. B246 (1990) 377;

I. Antoniadis, C. Munoz, M. Quiros Nucl. Phys. B397 (1993) 515;

I. Antoniadis, K. Benakli, M. Quiros Phys. Lett. B331 (1994) 313;

J. Lykken, Phys. Rev. D54, 3693 (1996);

I. Antoniadis, S. Dimopoulos and G. Dvali, Nucl. Phys. B516, 70 (1998);

N. Arkani-Hamed, S. Dimopoulos and G. Dvali, Phys. Lett. B429, 263 (1998);

J. Lykken, L. Randall, "The Shape of gravity," hep-th/9908076.

[2] W. A. Bardeen, R. B. Pearson and E. Rabinovici, Phys. Rev. D 21, 1037 (1980).

[3] B. W. Lee, C. Quigg and H. B. Thacker, Phys. Rev. D 16, 1519 (1977).

[4] M. Bando, T. Kugo and K. Yamawaki, Phys. Rept. 164 (1988), 217; Nucl. Phys. B259, (1985) 493; M. Bando, T. Kugo, S. Uehara and K. Yamawaki, Phys. Rev. Lett., 54, (1985), 1215; M. Bando, T. Fujiwara and K. Yamawaki, Prog. Theor. Phys., 79, (1988), 1140.

[5] K.R. Dienes, E. Dudas and T. Gherghetta, Phys. Lett. B436, 55 (1998);

K.R. Dienes, E. Dudas and T. Gherghetta, Nucl. Phys. B537, 47 (1999); see also: hep-ph/9807522;

[6] M. Dine and N. Seiberg, Phys. Lett. 162B, 299 (1985);

H.-C. Cheng, B. A. Dobrescu and C. T. Hill, "Electroweak symmetry breaking by extra dimensions", hep-ph/0004072 and hep-ph/9912343;

H.-C. Cheng, B. A. Dobrescu and C. T. Hill, Nucl.Phys. B573 597, (2000);

N. Arkani-Hamed, H.-C. Cheng, B. A. Dobrescu and L. J. Hall", "Self-breaking of the standard model gauge symmetry", hep-ph/0006238';

N. Arkani-Hamed and M. Schmaltz, "Hierarchies without symmetries from extra dimensions," hep-ph/9903417.

[7] T. Eguchi and H. Kawai, Phys. Rev. Lett. 48, 1063 (1982).

[8] E. Poqqio, H. Quinn and S. Weinberg, Phys. Rev. D13 1958, (1976); D. Ross, Nucl.Phys. B140 1, (1978).

[9] G. Dvali, I. I. Kogan and M. Shifman, Phys. Rev. D 62, 106001 (2000); C. T. Hill and P. Ramond, Nucl. Phys. B 596, 243 (2001). 
[10] C.T. Hill, S. Pokorski, J. Wang, work in progress.

[11] N. Arkani-Hamed, A. G. Cohen and H. Georgi, hep-th/0104005. 